\section{Editorial}

Check for updates

\section{OPEN ACCESS}

Received: Jan 6, 2019

Accepted: Jan 15, 2019

Address for Correspondence:

In-Jeong Cho, MD

Division of Cardiology, Severance

Cardiovascular Hospital, Yonsei University

College of Medicine, 50-1 Yonsei-ro,

Seodaemun-gu, Seoul 03722, Korea.

E-mail: injeong.md@gmail.com

Copyright (c) 2019 Korean Society of

Echocardiography

This is an Open Access article distributed under the terms of the Creative Commons Attribution Non-Commercial License (https:// creativecommons.org/licenses/by-nc/4.0/) which permits unrestricted non-commercial use, distribution, and reproduction in any medium, provided the original work is properly cited.

ORCID iDs

In-Jeong Cho iD

https://orcid.org/0000-0002-1209-5129

\section{Conflict of Interest}

The authors have no financial conflicts of interest.

\title{
Unraveling the Mechanism of Cardiac Remodeling in Overloaded Heart: From Experiment to Theory
}

\section{In-Jeong Cho}

Division of Cardiology, Severance Cardiovascular Hospital, Yonsei University College of Medicine, Seoul, Korea

- See the article "Differential Transcriptome Profile and Exercise Capacity in Cardiac Remodeling by Pressure Overload versus Volume Overload" in volume 27 on page 50.

In cardiovascular disease, the left ventricle (LV) demonstrates a plasticity response called remodeling. ${ }^{1)}$ This remodeling process includes a complex series of transcriptional, signaling, electrophysiological, structural, and functional alterations occurring within the cardiac myocyte, as well as other components of the LV, including fibroblasts, vascular smooth muscle cells, endothelial cells, and leukocytes. ${ }^{2)}$

Cardiovascular diseases associated with pressure overload (PO) and volume overload (VO) produce morphologically and functionally distinct forms of LV remodeling. ${ }^{3)} \mathrm{PO}$, common in hypertension or aortic stenosis, results in concentric LV hypertrophy, whereas VO, seen in aortic regurgitation or mitral regurgitation, results in eccentric LV hypertrophy. Concentric LV hypertrophy is associated with increased LV wall thickness, whereas eccentric LV hypertrophy is characterized by LV chamber dilation, although there is a general increase in the overall size of cardiomyocytes in both remodeling processes. For long, it has been suggested that $\mathrm{PO}$ and $\mathrm{VO}$ are distinct biological phenomena mediated through different mechanisms, as shown by the different role of catecholamine in the hypertrophic process and some drugs that satisfactorily attenuate PO-induced hypertrophy but not VO-induced hypertrophy. ${ }^{4}$ Therefore, structural, functional, and molecular adaptations of the heart differ under $\mathrm{PO}$ and VO. You et al. ${ }^{5)}$ recently showed that the molecular phenotypes associated with $\mathrm{PO}$ and $\mathrm{VO}$ are different. They reported that the MAP kinase family-, $\beta$-arrestin-2-, Akt-, and $\mathrm{Ca}^{2+}$-related signaling pathways were markedly activated in a $\mathrm{PO}$ model but mildly upregulated or unchanged in a VO model, even when the degree of cardiac hypertrophy was similar between the two groups.

In this issue of the journal, Kim et al. ${ }^{6}$ (compared the gene expression profiles, diastolic function, exercise capacity, and cardiac fibrosis burden in the hypertrophied myocardium of rats subjected to $\mathrm{PO}$ and VO. Moreover, they set up a new animal model of combined $\mathrm{PO}$ and VO by suprarenal aortic constriction followed by surgical creation of mitral regurgitation with a 2-week interval. The authors found that a rat model with isolated diastolic dysfunction derived from $\mathrm{PO}$ demonstrated consistent results of enhanced fibrosis on gene and pathologic analyses, suggesting that the predominant mechanism was cardiac fibrosis, and that cardiac fibrosis caused diastolic dysfunction and impaired exercise capacity. This characteristic of a PO model was distinct from that of a VO model, which showed 
better exercise capacity, less cardiac fibrosis, and a different genetic pathway of enriched inflammatory response. Therefore, prudent consideration should be given when mechanistic studies are performed, depending on whether PO or VO occurs in the specific cardiovascular disease targeted. These findings also imply that different pharmaceutical interventions are required for $\mathrm{LV}$ hypertrophy depending on the contribution of $\mathrm{PO}$ or VO.

One of the interesting findings of their study was that a combined PO and VO model showed better exercise capacity and less cardiac fibrosis compared to a simple PO model. The authors explained this phenomenon as a switch-on for the gene according to activation time. They concluded that timely intervention of switch-on for the genes toward favorable directions would attenuate LV fibrosis and prevent diastolic dysfunction that developed under PO. This theory might play an important role in future mechanistic studies and pharmacological interventions to attenuate the hazardous impact of $\mathrm{PO}$ on the LV myocardium. Further mechanistic and pharmacological studies are required to investigate stimulus-specific diversity in the signaling pathways and develop novel intervention methods to alter adverse pathways by changing overloading conditions. Above all, experiments with animal models would be the first step to increasing our understanding of the pathologic mechanisms in LV hypertrophy and to developing a novel theory to overcome and prevent adverse LV remodeling in an overloaded heart.

\section{REFERENCES}

1. Hill JA, Olson EN. Cardiac plasticity. N Engl J Med 2008;358:1370-80. PUBMED | CROSSREF

2. Burchfield JS, Xie M, Hill JA. Pathological ventricular remodeling: mechanisms: part 1 of 2. Circulation 2013;128:388-400. PUBMED | CROSSREF

3. Lorell BH, Carabello BA. Left ventricular hypertrophy: pathogenesis, detection, and prognosis. Circulation 2000;102:470-9. PUBMED | CROSSREF

4. Rossi MA, Carillo SV. Cardiac hypertrophy due to pressure and volume overload: distinctly different biological phenomena? Int J Cardiol 1991;31:133-41. PUBMED | CROSSREF

5. You J, Wu J, Zhang Q, et al. Differential cardiac hypertrophy and signaling pathways in pressure versus volume overload. Am J Physiol Heart Circ Physiol 2018;314:H552-62. PUBMED

6. Kim KH, Kim HM, Park JS, Kim YJ. Differential transcriptome profile and exercise capacity in cardiac remodeling by pressure overload versus volume overload. J Cardiovasc Imaging 2019;27:50-63. CROSSREF 